\title{
Order of draw practices in venous blood sampling at clinical biochemistry departments in the Danish health care system
}

Jacobsen, Katja Kemp; Brandt, Ida; Christensen, Anne Vindahl; Rimsø, Bjørk Anine; Krøier, Camilla Julie; Sørensen, Michelle; Smith, Julie; Jensen, Kathrine Overgaard Foss; Larsen, Jeppe Madura

\section{Published in:}

Clinical Biochemistry

Link to article, DOI:

10.1016/j.clinbiochem.2018.04.020

Publication date:

2018

Document Version

Peer reviewed version

Link back to DTU Orbit

Citation (APA):

Jacobsen, K. K., Brandt, I., Christensen, A. V., Rimsø, B. A., Krøier, C. J., Sørensen, M., Smith, J., Jensen, K. O. F., \& Larsen, J. M. (2018). Order of draw practices in venous blood sampling at clinical biochemistry departments in the Danish health care system. Clinical Biochemistry, 56, 113-116.

https://doi.org/10.1016/j.clinbiochem.2018.04.020

\section{General rights}

Copyright and moral rights for the publications made accessible in the public portal are retained by the authors and/or other copyright owners and it is a condition of accessing publications that users recognise and abide by the legal requirements associated with these rights.

- Users may download and print one copy of any publication from the public portal for the purpose of private study or research.

- You may not further distribute the material or use it for any profit-making activity or commercial gain

- You may freely distribute the URL identifying the publication in the public portal 


\section{Accepted Manuscript}

Order of draw practices in venous blood sampling at clinical biochemistry departments in the Danish health care system

Katja Kemp Jacobsen, Ida Brandt, Anne Vindahl Christensen, Bjørk Anine Rimsø, Camilla Julie Krøier, Michelle Sørensen, Julie Smith, Kathrine Overgaard Foss Jensen, Jeppe Madura

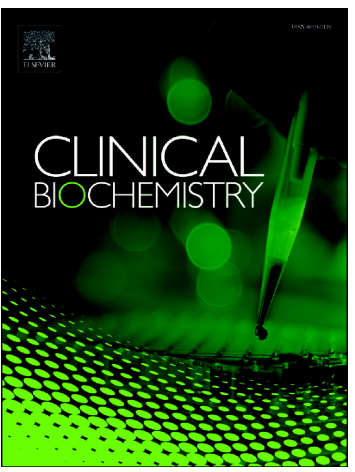
Larsen

PII: S0009-9120(17)31185-2

DOI: doi:10.1016/j.clinbiochem.2018.04.020

Reference: CLB 9772

To appear in: Clinical Biochemistry

Received date: 4 December 2017

Revised date: 18 April 2018

Accepted date: 19 April 2018

Please cite this article as: Katja Kemp Jacobsen, Ida Brandt, Anne Vindahl Christensen, Bjørk Anine Rimsø, Camilla Julie Krøier, Michelle Sørensen, Julie Smith, Kathrine Overgaard Foss Jensen, Jeppe Madura Larsen, Order of draw practices in venous blood sampling at clinical biochemistry departments in the Danish health care system. The address for the corresponding author was captured as affiliation for all authors. Please check if appropriate. $\mathrm{Clb}$ (2017), doi:10.1016/j.clinbiochem.2018.04.020

This is a PDF file of an unedited manuscript that has been accepted for publication. As a service to our customers we are providing this early version of the manuscript. The manuscript will undergo copyediting, typesetting, and review of the resulting proof before it is published in its final form. Please note that during the production process errors may be discovered which could affect the content, and all legal disclaimers that apply to the journal pertain. 


\section{Short Communication}

\section{Order of draw practices in venous blood sampling at clinical biochemistry departments in the Danish health care system}

Katja Kemp Jacobsen ${ }^{1}$, Ida Brandt ${ }^{1}$, Anne Vindahl Christensen ${ }^{1}$, Bjørk Anine Rims $\emptyset^{1}$, Camilla Julie Krøier ${ }^{1}$, Michelle Sørensen ${ }^{1}$, Julie Smith ${ }^{1}$, Kathrine Overgaard Foss Jensen ${ }^{2}$, Jeppe Madura Larsen $^{1,3}$

${ }^{1}$ Department of Technology, Faculty of Health and Technology, University College Copenhagen, , Copenhagen, Denmark

${ }^{2}$ Department of Clinical Biochemistry, Rigshospitalet, Copenhagen University Hospital, Denmark

${ }^{3}$ National Food Institute, Technical University of Denmark, Lyngby, Denmark Keywords: venous blood sampling, order of blood draw, phlebotomy, preanalytical phase, preanalytical quality, inter-laboratory variation

Corresponding Author: Katja Kemp Jacobsen, Department of Technology, Faculty of Health and Technology, University College Copenhagen, Denmark. E-mail: katj@phmetropol.dk. Phone: +45 72457800 


\section{Abstract}

\section{Background}

Deviation in blood collection procedures is a central source of preanalytical variation affecting overall analytical and diagnostic precision. The order of draw of venous sampling is suspected to affect analytical results, in particular for coagulation analysis. Here we compare the procedures in venous blood sampling among clinical biochemistry departments to assess the uniformity of order of blood draw and adherence to international guidelines in the Danish health care system.

\section{Methods}

We collected venous order of draw procedures from 49 clinical biochemistry departments at 22 public hospitals in Denmark. Procedures were compared to the international guidelines fromthe Clinical Laboratory Standards Institute (CLSI) and World Health Organization (WHO), and assessed in relation to department ISO 15189:2012 accreditation.

\section{Results}

We observed seven different order of draw procedures related to citrate, serum, heparin, and EDTA tubes, and the use of discard tubes in relation to coagulation assays. 31 departments (63.3 $\%$ ) were found to adhere to CLSI and WHO guidelines. A majority of departments instructs the use of discard tubes before collection for coagulation assays in citrate tubes (44 departments; $89.8 \%$ ). The citrate tube was the first sample tube to be drawn for most departments ( 35 departments; 75.5 $\%$ ); and the preferred order of non-citrate tubes was serum-heparin-EDTA (36 departments; 73.5 $\%)$. Adherence to the CLSI and WHO guidelines was not associated with department ISO 15189:2012 accreditation ( $p=0.57$ ).

\section{Conclusions}

Venous order of draw procedures are diverse at Danish clinical biochemistry departments and show moderate adherence to international guidelines. 


\section{Introduction}

The majority of errors (46.0\%-68.2\%) in the total process of laboratory medicine occur in the preanalytical phase (1-4). An important step in this phase is venous blood sampling where the order of draw has been advocated as a potential source of errors. A recent systematic review of the available literature by the European Federation for Clinical Chemistry and Laboratory Medicine (EFLM) Working group for Preanalytical Phase (5) supported the importance of the correct order of draw to prevent contamination due to additive carryover.

Evidence-based guidelines have been developed by the Clinical Laboratory Standards Institute (CLSI) (10) and World Health Organization (WHO) (11). However, Danish compliance to international guidelines and inter-laboratory variation is currently not known. Therefore, the aim of this study was to assess the uniformity on venous order of blood draw and adherence to CLSI and WHO guidelines in the Danish health care system. In Denmark, order of draw decisions are not necessarily made at hospital level, and each clinical biochemistry departments follow their own local procedureson venous blood draw. Thus, the uniformity in procedures was analyzed at department level. Furthermore, we assessed whether departmental accreditation by ISO 15189:2012 (Medical laboratories - Requirements for quality and competence) was associated with adherence to international guidelines.

\section{Materials and methods}

We systematically collected venous order of draw procedures from clinical biochemistry departments that perform blood sampling in all public hospitals in Denmark. We did not include health centers and psychiatric departments. The collection of procedures was performed in the period from February to April 2017. If a hospital had more than one clinical biochemistry department, then procedures were obtained for each department independent of whether or not the department had different procedures or management. If available, procedureswere retrieved from hospital websites, otherwise departments were contacted by mail or phone. We also collected data on whether or not the department had received ISO 15189:2012 accreditation, which specifies requirements for quality and competence in medical laboratories (12).

We recorded and compared the order of draw for citrate, serum, heparin and EDTA tubes, as well as the use of discard tubes in relation to coagulation assays. No distinction was made as to whether or not the proceduresrecommended the use of specific clot activators or gels in the specific tube types. The collected Danish procedureswere compared to the guidelines recommended by CLSI and WHO $(10,11)$. 
The association between department ISO 15189:2012 accreditation and the adherence to CLSI and WHO guidelines $(10,11)$ was analyzed by chi ${ }^{2}$ test using MS Excel 2010. 
Table 1 Venous order of draw guidelines from the Clinical Laboratory Standards Institute (CLSI) and the World Health Organization (WHO) $(10,11)$ and procedures in clinical biochemistry departments $(n=49)$ from 22 public hospitals in Denmark obtained between February to April 2017; for the order of draw of citrate tubes, serum tubes, heparin tubes and EDTA tubes, and the use of discard tubes related to coagulation tubes.

\begin{tabular}{|c|c|c|c|c|c|c|c|}
\hline \multirow{2}{*}{\multicolumn{3}{|c|}{ Guidelines }} & \multicolumn{5}{|c|}{ Recommended order of blood draw } \\
\hline & & & $\begin{array}{l}\text { Use of } \\
\text { discard tube }\end{array}$ & 1st tube & 2nd tube & 3rd tube & $\begin{array}{l}\text { 4th } \\
\text { tube }\end{array}$ \\
\hline $\begin{array}{l}\text { CLSI (10), } \\
\text { WHO (11) }\end{array}$ & & 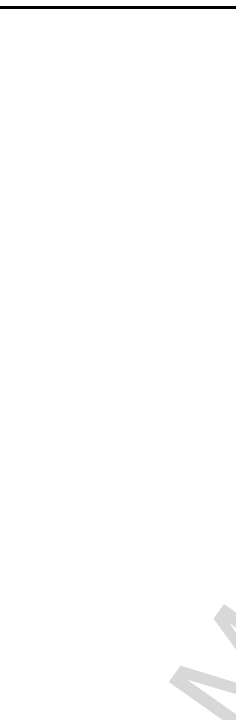 & $\begin{array}{l}\text { When } \\
\text { coagulation } \\
\text { tubes are } \\
\text { collected as } \\
\text { the first or the } \\
\text { only tube } \\
\text { Using a } \\
\text { straight } \\
\text { needle: Not } \\
\text { for INR and } \\
\text { PT } \\
\text { Using a } \\
\text { winged blood } \\
\text { collection set: } \\
\text { Alw ays. }\end{array}$ & Citrate & Serum & Heparin & EDTA \\
\hline Procedures & & 2 & & & & & \\
\hline $\begin{array}{l}\text { Departments } \\
\text { grouped by } \\
\text { procedure) }\end{array}$ & $\begin{array}{l}\text { De partments (\% of } \\
\text { total, } n=49 \text { ) }\end{array}$ & $\begin{array}{l}\text { Achieved } \\
\text { department } \\
\text { accre ditation of } \\
\text { ISO 15189:2012) } \\
\text { (\% of total) }\end{array}$ & $\begin{array}{l}\text { Use of } \\
\text { discard tube }\end{array}$ & 1st tube & 2nd tube & 3rd tube & $\begin{array}{l}\text { 4th } \\
\text { tube }\end{array}$ \\
\hline Group 1 & $31(63.3)$ & $23(74.2)$ & $\begin{array}{l}\text { Alw ays, if } \\
\text { coagulation } \\
\text { tubes are } \\
\text { collected }\end{array}$ & Citrate & Serum & Heparin & EDTA \\
\hline Group 2 & $6(12.2)$ & $3(50.0)$ & $\begin{array}{l}\text { Alw ays, if } \\
\text { coagulation } \\
\text { tubes are } \\
\text { collected }\end{array}$ & No spec & er for the re & aining tubes & \\
\hline Group 3 & $4(8.2)$ & $4(100.0)$ & Not specified & Heparin & Citrate & EDTA & Serum \\
\hline Group 4 & $3(6.1)$ & $0(0.0)$ & Alw ays & Citrate & Heparin & Serum & EDTA \\
\hline Group 5 & $2(4.1)$ & $2(100.0)$ & Alw ays & Citrate & Serum & Heparin & EDTA \\
\hline Group 6 & $2(4.1)$ & $2(100.0)$ & $\begin{array}{l}\text { Alw ays, if } \\
\text { coagulation } \\
\text { tubes are } \\
\text { collected } \\
\text { (except for }\end{array}$ & Serum & Citrate & Heparin & EDTA \\
\hline
\end{tabular}




\begin{tabular}{|l|l|l|l|l|l|l|l|}
\hline & & & INR or PT) & & & & \\
\hline Group 7 & $1(2.0)$ & 1 & Never & Citrate & Serum & Heparin & EDTA \\
\hline
\end{tabular}

Prothrombin time (PT), International normalized ratio (INR).

\section{Results}

\section{Procedures collected}

Venous blood order of draw procedures were collected from 49 clinical biochemistry departments at 22 public hospitals within the Danish public health care system.

\section{Order of draw}

We observed seven different variations of order of draw procedures related to citrate, serum, heparin, and EDTA tubes, and the use of discard tubes related to coagulation assays (Tabel 1). The most frequent order of draw procedurecovering 31 departments (63.3\%) were in line with the CLSI and WHO guidelines. However, the remaining 18 departments $(12.2 \%)$ were found to have diverse procedureswith six different order of draw policies. 43 of 49 departments (87.8\%) gave proceduresfor order of draw with 37 (75.5\%), 4 (8.2\%), and 2 (4.1\%) departments recommending the first draw to be citrate, heparin, or serum tubecontainers, respectively (Figure 1A). When addressing the order of draw for non-citrate tubes, the most frequent order was serum-heparin-EDTA for 36 departments (73.5\%), followed by heparin-serumEDTA and heparin-EDTA-serum for 4 (8.16\%) and 3 (6.12\%) departments, respectively. Use of discard tube Procedures from 39 departments $(79.6 \%)$ specifically stated that discard tubes should be used before drawing citrate tubes; however, two of these departments (4.1\%) specifically excluded discard tubes when INR and PT assays were ordered (Table 1, and Figure 1B). The remaining procedures recommended to always (5 departments; $10.2 \%$ ), to never (1 departments; $2.0 \%$ ), or did not specify (4 departments; $8.2 \%$ ) the use of a discard tube. In total, the procedures from 44 departments $(89.8 \%)$ de facto recommended the use of a discard tube before drawing a citrate tube.

\section{Department accreditation}

In total 35 departments (71.4\%) were accredited by ISO 15189:2012 (Table 1). Of the accredited departments 23 out of 31 (74.2\%) had procedures in line with the CLSI (10) and WHO guidelines (11). Within the group of departments with diverse procedures 12 of 18 (67.0\%) were accredited. We found no association between department accreditation and adherence to the international CLSI and WHO guidelines ( $p=0.57$, chi ${ }^{2}$-test).

\section{Discussion}

We observed seven different variations of order of blood draw procedures from the clinical biochemistry departments queried. Almost two thirds of the departments were compliant to the guidelines from the CLSI and WHO $(10,11)$. 
The translation of evidence-based guidelines of order of draw to clinical practice seems manageable and straightforward. The order of draw guidelines are easy to follow, and are not more time-consuming compared to an established practice. However, the moderate adherence to international guidelines of order of draw at Danish clinical biochemistry departments was unexpected given the ease of following. Considerable factors might be influencing the adoption in practice such as the management of department's unawareness of the guidelines, old traditions, insufficient time and work pressure. Another reasonable explanation is the insufficient evidence of the outcome not adopting international guidelines. The order of draw is a potential preanalytical contributor to diagnostic errors in the total testing phase, and case studies $(8,9)$ indicate that some test errors goes undetected (13). However, increased evidence about the preanalytical errors and subsequently patient-related consequences might promote better compliance to international guidelines regarding the order of draw (14). It is important to underline that we only report what is described in the procedures collected from the departments. Some steps in the order of draw process may be perceptive assumed by staff within a department and thereby not described explicitly, such as the case of group 2 in the procedures.

Furthermore, we cannot be sure that the procedures on the hospitals web sides are up to date.

CLSI (10) recommends using a discard tube to be prime the tubing drawn when using a winged needleblood collection set, thereby ensuring a proper anticoagulant to blood ratio Several studies (15-20) have shown that PT/INR, APTT and some specialized coagulation assay results are not affected if tested on the first tube collected, without the use of a discard tube. These results dispel the misperception that a discard tube should be collected to avoid the effect of thromboplastin. The discard tube is used to ensure maintenance of the proper ratio in the first tube, as prior studies does have conflicting conclusions due to the impact of under filling on coagulation assay results $(15-16,21)$. .The use of a discard tube is costly, and it would be interesting if future studies could evaluate thebold suggestions not using a discard tube.

Drawing blood is regularly performed outside the laboratory for instance at bedside, at surgical procedures, or may be collected and sent from the local general practitioner. Outside the laboratories, personnel with different professional backgrounds often carry out phlebotomy. These different settings with potential discrepancy in procedures will add variation and increase the risk of errors. It is even suggested that professional phlebotomists are only compliant to the local guidelines in $8.1 \%$ of all venous blood collections (12). To ensure the preanalytical quality in clinical laboratory practice it is crucial that every step in the total testing process is performed using evidence-based procedures. National and international harmonization may improve preanalytical 
variation in inter-hospital clinical studies and practice. It is an eye-opener to identify this moderate adherence to evidence-based guidelines and such diversity within a small country like Denmark. Also, the accreditation standards does not seem to emphasize the need for standardized guidelinebased order of draw procedures, seem there is no consistency in which laboratories achieved accreditation with in regards to neither order of draw nor the use of discard tube. Implementation of correct and standardized procedures inside and outside clinical biochemistry departments will presumably reduce the risk of test result errors, and furthermore increase the comparably of test results from one facility to another. Therefore, we recommend that higher compliance to international guidelines concerning the order of draw should be prioritized in the future. Hopefully, the upcoming recommendation from the European Federation of Clinical Chemistry and Laboratory Medicine (EFLM) Working group for Preanalytical Phase (WG-PRE) (22) will encourage professionals throughout Europe to implement evidence-based guidelines of blood collection practices.

\section{Author contributions}

Katja Kemp Jacobsen and Jeppe Madura Larsen conceived and designed the study, analyzed data, and wrote the manuscript. Ida Brandt, Anne Vindahl Christensen, Bjørk Anine Rimsø, Camilla Julie Krøier and Michelle Sørensen collected procedures and compiled order-of-draw data. Julie Smith and Kathrine Overgaard Foss Jensen provided important intellectual contributions to the study and writing of manuscript.

\section{Acknowledgements}

The authors would like to thank medical laboratory scientist students, Mathias Bech-Thomasen, Mahamed Y. Hassan, Mona Al-Rashedi, and Tiba Younes for assisting in the collection of procedures. 


\section{References}

1. Carraro P, Plebani M. Errors in a stat laboratory: types and frequencies 10 years later. Clin Chem. 2007;53(7):1338-1342.

2. Kalra J. Medical errors: impact on clinical laboratories and other critical areas. Clin Biochem. 2004;37(12):1052-1062.

3. Plebani M, Laposata M, Lundberg GD. The brain-to-brain loop concept for laboratory testing 40 years after its introduction. Am J Clin Pathol. 2011;136(6):829-833.

4. Plebani M, Carraro P. Clin Chem. Mistakes in a stat laboratory: types and frequency. 1997;43(8 Pt 1):1348-1351.

5. Cornes M, van Dongen-Lases E, Grankvist K, lbarz M, Kristensen G, Lippi G, Nybo M, Simundic AM; Working Group for Preanalytical Phase (WG-PRE), European Federation of Clinical Chemistry and Laboratory Medicine (EFLM). Order of blood draw: Opinion Paper by the European Federation for Clinical Chemistry and Laboratory Medicine (EFLM) Working Group for the Preanalytical Phase (WG-PRE). Clin Chem Lab Med. 2017;1;55(1):27-31.

6. Fukugawa Y, Ohnishi H, Ishii T, Tanouchi A, Sano J, Miyawaki H, Kishino T, Ohtsuka K, Yoshino $\mathrm{H}$, Watanabe T. Effect of carryover of clot activators on Citrate assays during phlebotomy. Am J Clin Pathol. 2012;137(6):900-903.

7. Indevuyst C, Schuermans W, Bailleul E, Meeus P. The order of draw: much ado about nothing? Int J Lab Hematol. 2015;37(1):50-55.

8. Cornes MP, Ford C, Gama R. Spurious hyperkalaemia due to EDTA contamination: common and not always easy to identify. Ann Clin Biochem. 2008;45(Pt 6):601-603.

9. Cornes MP, Davidson F, Darwin L, Gay C, Redpath M, Waldron JL, Ford C, Gama R. Clin Lab. Multi-centre observational study of spurious hyperkalaemia due to EDTA contamination.

2010;56(11-12):597-599.

10. CLSI, Collection of Diagnostic Venous Blood Specimens. 7th ed. CLSI standard GP41. Wayne, PA: Clinical Laboratory Standards Institute, 2017.

11. WHO guidelines on drawing blood: best practices in phlebotomy. Available at: http://whqlibdoc.who.int/publications/2010/9789241599221_eng.pdf (accessed 29.01.17).

12. ISO 15189:2012. Medical laboratories -- Requirements for quality and competence. Available at: https://www.iso.org/standard/56115.html (accessed 13.02.18).

13. Simundic AM, Church S, Cornes MP, Grankvist K, Lippi G, Nybo M, Nikolac N, van DongenLases E, Eker P, Kovalevskaya S, Kristensen GB, Sprongl L, Sumarac Z. Compliance of blood sampling procedures with the CLSI H3-A6 guidelines: An observational study by the European 
Federation of Clinical Chemistry and Laboratory Medicine (EFLM) working group for the preanalytical phase (WG-PRE). Clin Chem Lab Med. 2015;53(9):1321-1331.

14. Grol R, Grimshaw J. From best evidence to best practice: effective implementation of change in patients' care. Lancet. 2003;11;362(9391):1225-1230.

15. Adcock DM, Kressin DC, Marlar RA. Minimum specimen volume requirements for routine Citrate testing: dependence on Citrateconcentration. Am J Clin Pathol. 1998;109(5):595-599.

16. Raijmakers MT, Menting CH, Vader HL, van der Graaf F. Collection of blood specimens by venipuncture for plasma-based coagulation assays: necessity of a discard tube. Am J Clin Pathol. 2010 Feb;133(2):331-335.

17. Yawn BP, Loge C, Dale J. Prothrombin time: one tube or two. Am J Clin Pathol. 1996;105(6):794-797.

18. Gottfried EL, Adachi MM. Prothrombin time and activated partial thromboplastin time can be performed on the first tube. Am J Clin Pathol. 1997;107(6):681-683.

19. Bamberg R, Cottle JN, Williams JC. Effect of drawing a discard tube on PT and APTT results in healthy adults. Clin Lab Sci. 2003;16(1):16-19.

20. Favaloro EJ, Lippi G, Raijmakers MT, Vader HL, van der Graaf F. Discard tubes are sometimes necessary when drawing samples for hemostasis. Am J Clin Pathol. 2010;134(5):851. 21. Kim H, Kim Y, Lee HK. Influence of Preanalytical Variables on Prothrombin Time, Activated Partial Thromboplastin Time, and Fibrinogen. Clin Lab. 2015;61(9):1337-1340.

22. Simundic AM, Bolenius K, Cadamuro J, Church S, Cornes MP, van Dongen-Lases EC, Eker P, Erdeljanovic T, Grankvist K, Guimaraes JT, Hoke R, lbarz M, Ivanov H, Kovalevskaya S, Kristensen GBB, Lippi G, von Meyer A, Nybo M, De la Salle B, Seipelt C, Sumarac Z, Vermeersch $P$, on behalf of the Working Group for Preanalytical Phase (WG-PRE), European Federation of Clinical Chemistry and Laboratory Medicine (EFLM). EFLM recommendation of venous blood sampling v 1.1, October 2017. Available at: http://www.dskb.dk/Messages/Inbox/GetFile.aspx?id=7707 (accessed 27.02.18). 
Figure 1a-bThe distribution of procedures of order of draw according to the use of a first draw and use of a discard tube. Achieved from 49 clinical biochemistry departments, covering 22 public hospitals in Denmark.

Figure 1a

\section{First tube drawn}

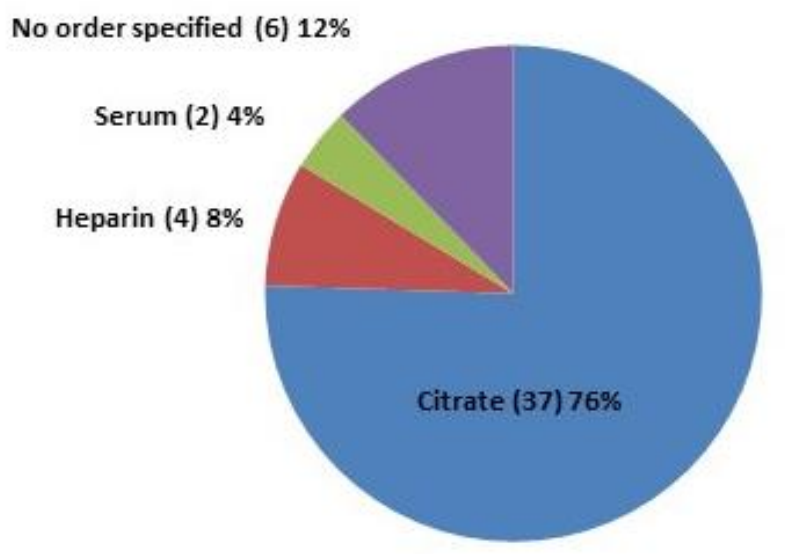

\section{Use of discard tube}

Not specified $8 \% \quad$ Never $2 \%$

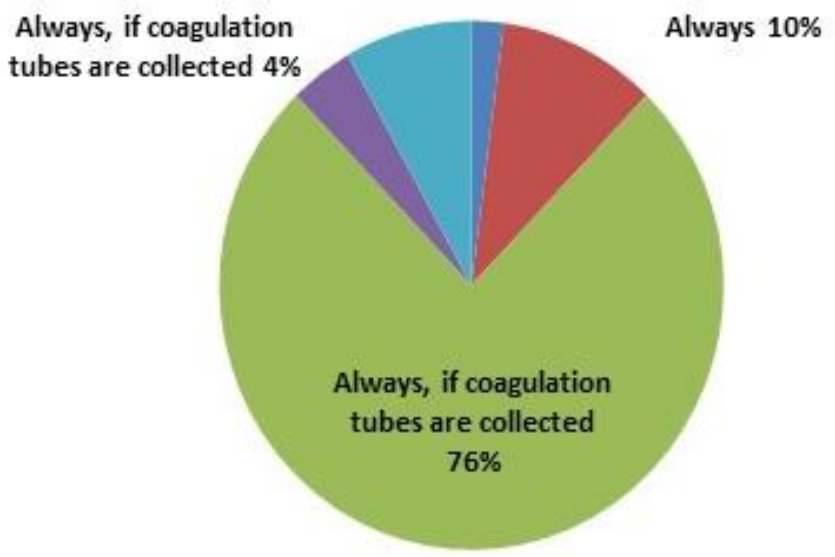

Figure $1 b$ 


\section{Highlights}

- We observed seven different variations of order of blood draw procedures from the clinical biochemistry departments in Denmark.

- Only two thirds of the procedures were compliant to the evidence-based guidelines from the Clinical Laboratory Standards Institute (CLSI) and the World Health Organization (WHO).

- Evidence-based and standardized order of blood draw procedures at all clinical biochemistry departments will presumably reduce the risk of test result errors, and furthermore increase the comparably of test results from one facility to another.

- We recommend that higher compliance to evidence-based guidelines concerning the order of draw should be prioritized in the future. 